\title{
Structural basis for broad anti-phage immunity by DISARM
}

Jack P. K. Bravo ${ }^{*}$, Cristian Aparicio-Maldonado ${ }^{2,3 *}$, Franklin L. Nobrega ${ }^{4}$, Stan J. J. Brouns $^{2,3 \dagger}$, and David W. Taylor ${ }^{1,5-7 \dagger}$

${ }^{1}$ Department of Molecular Biosciences, University of Texas at Austin, Austin, TX, 78712, USA

2Department of Bionanoscience, Delft University of Technology, Delft, The Netherlands

${ }^{3}$ Kavli Institute of Nanoscience, Delft, The Netherlands

${ }^{4}$ School of Biological Sciences, University of Southampton, SO17 1BJ, Southampton, United Kingdom

Institute for Cellular and Molecular Biology, University of Texas at Austin, Austin, TX, 78712, USA

${ }^{6}$ Center for Systems and Synthetic Biology, University of Texas at Austin, Austin, TX, 78712, USA

${ }^{7}$ LIVESTRONG Cancer Institutes, Dell Medical School, Austin, TX, 78712, USA

*These authors contributed equally to this work.

†Correspondence to: S.J.J.Brouns@tudelft.nl (SJJB) and dtaylor@utexas.edu (DWT)

\begin{abstract}
In the evolutionary arms race against phage, bacteria have assembled a diverse arsenal of antiviral immune strategies. While the recently discovered DISARM (Defense Island System Associated with Restriction-Modification) systems can provide protection against a wide range of phage, the molecular mechanisms that underpin broad antiviral targeting but avoiding autoimmunity remain enigmatic. Here, we report cryo-EM structures of the core DISARM complex, DrmAB, both alone and in complex with an unmethylated phage DNA mimetic. These structures reveal that DrmAB core complex is autoinhibited by a trigger loop (TL) within DrmA and binding to DNA substrates containing a 5' overhang dislodges the TL, initiating a long-range structural rearrangement for DrmAB activation. Together with structure-guided in vivo studies, our work provides insights into the mechanism of phage DNA recognition and specific activation of this widespread antiviral defense system.
\end{abstract}




\section{Introduction}

The threat of bacteriophage has driven bacteria to evolve a myriad of antiviral defense systems, capable of targeting phage at various stages of infection and replication ${ }^{1,2}$. The first lines of antiviral defense are typically rapid, broad, innate immune responses that include preventing phage adsorption ${ }^{3}$, degrading foreign DNA using restriction-modification (RM) systems $^{4}$, or ultimately host cell destruction through abortive infection (Abi) systems ${ }^{1}$. Adaptive immune responses (including CRISPR-Cas systems) provide a highly-specific and long-term antiviral protection ${ }^{5,6}$. A number of recently discovered anti-phage systems have expanded the arsenal of defense tools used by microbes in the arms race against phage ${ }^{7-9}$.

RM systems are the most highly abundant bacterial defense system, found in $\sim 75 \%$ of all bacterial genomes ${ }^{10}$. They often consist of DNA methyltransferase, restriction endonuclease, and target recognition modules. The vast majority rely on sequence-specific DNA methylation, which is recognized by and rapidly degraded by specific nucleases, allowing for self-versusnon-self discrimination to avoid autoimmunity. However, phage have adapted to evade such systems by either evolving to lack the restriction site sequences required for DNA cleavage, or carrying their own epigenetic modification to prevent being targeted ${ }^{4,11}$.

The largely uncharacterized DISARM (Defense Island System Associated with RestrictionModification) systems are widespread in bacteria ${ }^{7}$. The DISARM operon typically contains a DNA methyltransferase (DrmMI and/or DrmMII that methylate adenine and cytosine, respectively) along with a helicase (DrmA), a DUF (domain of unknow function)1998containing protein (DrmB), a phospholipase D (PLD) domain nuclease (DrmC), amongst additional auxiliary genes ${ }^{7}$ (Fig. 1a). DUF1998 domains are common in various defense systems including Druantia ${ }^{8}$ and $\mathrm{Dpd}^{12}$, suggesting a common role in antiphage activity. While the presence of methyltransferase and nuclease genes within the DISARM operon hints that this system behaves akin to other RM systems, DrmC appears to be non-essential for DISARM activity, and DISARM can still restrict phage that lack methylation target sequences ${ }^{13}$. Thus, the molecular mechanisms underlying phage targeting remain unclear.

Here, we report cryo-EM structures of DrmA:DrmB (DrmAB) in the presence or absence of target DNA, revealing the arrangement of conserved RecA helicase domains relative to the DUF1998 domain. In vivo studies of structure-guided DrmAB mutants demonstrate that ATP hydrolysis, DNA binding, and DrmAB heterodimer formation are essential for phage targeting by DISARM. We observe that DrmA contains an unstructured trigger loop that partially occludes the DNA-binding surface on the complex. This loop limits DNA binding for dsDNA and allows DrmAB to discriminate between targets based on DNA structure rather than DNA sequence as a mechanism of self-versus-non-self-discrimination. DNA loading induces longrange conformational changes likely associated with DISARM activation. Our results elucidate how DISARM systems can provide broad antiphage activity, while avoiding autoimmune activation and reveal key molecular mechanisms that underpin rapid DISARM activation upon phage infection. 


\section{Results \\ Cryo-EM of DrmAB complex}

We purified the Serratia sp. SCBI (Serratia) DrmAB complex after co-expression of DrmA and DrmB in E. coli. SDS-PAGE analysis of the peak fraction from size-exclusion chromatography confirmed the presence of both DrmA and DrmB, in a 1:1 stoichiometry (Supplementary Fig. 1). To capture the DrmAB complex bound to a phage single-stranded DNA mimic, we incubated DrmAB with an unmethylated forked DNA substrate in the presence of ADP. DrmAB:ADP:DNA complex formation was confirmed by native electrophoretic mobility shift assays (Supplementary Fig. 1).

We used cryo-EM to investigate the architecture of the DrmAB:ADP:DNA complex. During data collection we observed that the complex adopts a preferred orientation in vitreous ice, which was ameliorated through collecting additional data at a $-30^{\circ}$ tilt. Multiple rounds of classification resulted in a 3D reconstruction of the complex at a nominal resolution of $2.8 \AA$ and focused 3D classification improved densities corresponding to DNA and the N-terminus of DrmB. Notably, we were able to separate DNA-bound from DNA-free particles through focused 3D classification, ultimately yielding 3D reconstructions of DrmAB:ADP:DNA and DrmAB:ADP at nominal resolutions of 3.3 and $3.4 \AA$, respectively (Supplementary Fig. 2). We built near-complete atomic models of both complexes ( $>98 \%$ of the $220 \mathrm{kDa}$ protein components), including seven nucleotides of DNA in the DrmAB:ADP:DNA complex.

\section{Architecture of DrmAB nucleoprotein complex}

The DrmAB complex exhibits a bi-lobed architecture, resembling a partially open clam shell (Fig. 1b \& c). The top lobe contains the RecA1 domain of DrmA, while the bottom lobe contains the DrmA RecA2 domain and the entirety of DrmB.

As expected for a superfamily 2 (SF2) helicase, DrmA contains tandem RecA modules (residues 600-1150) that form an active site for ATP-binding and hydrolysis and DNA loading. The N-terminal half of DrmA acts as a structural chassis, holding these two helicase motor domains in place, and forming an interface with DrmB. While RecA1 and RecA2 have high structural similarity to many other SF2 helicase domains (Z-score $>14$ for more than 10 other helicase structures ${ }^{14}$ ), no other regions of DrmA were found to have significant structural homology to known protein domains. The solvent-exposed interface between the tandem RecA domains forms the nucleotide-binding site, while the bound DNA straddles these domains within the heart of the complex (Fig. 1b \& c). Only four of the seven deoxynucleotides observed within our structure are solvent-exposed, with the remaining three bases deeply buried within the DrmA(RecA1,RecA2):DrmB interface.

DrmB contains a C-terminal DUF1998 module, a domain often enriched within bacterial genomic defense islands 7,15 . DUF1998 domain sits towards the back of the complex, predominantly buried within $\mathrm{DrmB}$, positioned towards the interface between the two RecA domains of DrmA. Like other proteins containing DUF1998 domains, DrmB contains the predicted zinc-coordinating four cysteine motif (C559,565,581,584, Supplementary Fig. 3) ${ }^{16,17}$. Surprisingly, we also observed an additional four putative coordinated metal ions at highly conserved sites within DrmB (Supplementary Fig. 3). While it is not possible to unambiguously determine the identity of these ions, based on the coordinating residues $\mathrm{Zn}^{2+}$ is a strong candidate ${ }^{18}$.

This raises the possibility that DrmAB may utilize these three highly conserved metal ion coordination sites to sense changes to cellular redox potential, triggered by environmental stresses such as phage infection, as has recently been demonstrated for the type III-A CRISPR system within Serratia ${ }^{19-22}$. Alternatively, these clusters may play a structural role, enabling proper folding of DrmB, as is the case for the DUF1998-containing StfH protein ${ }^{17}$. 
Multiple interaction surfaces contribute to the stabilization of the DrmAB complex, with a total buried surface area of $\sim 4,000 \AA^{2}$. Most notably, the C-terminus of DrmA (residues 1296-1319) wraps around the entirety of the N-terminus of DrmB (Fig. 1b,c), with a total buried surface area of $\sim 1850 \AA^{2}$. Additionally, the DrmB DUF1998 domain mediates $\sim 1230 \AA^{2}$ of surface contacts with DrmA (Fig. 1d). DUF1998 is positioned towards the interface between the two RecA domains of DrmA but makes limited contacts with both $(\sim 130$ and $\sim 110$ Å2 buried surface area with RecA1 and RecA2, respectively). RecA2 makes additional surface contacts with the $\mathrm{N}$-terminus of $\mathrm{DrmB}\left(482 \AA^{2}\right)$. Based on this network of inter-subunit interactions, we hypothesize that DUF1998-containing DrmB may act as a modulator of DrmA helicase activity. This is supported by the observation that DUF1998 domains are frequently associated with helicase domain-containing proteins, either as adjacent genes or as C-terminal fusions ${ }^{15}$.

\section{DNA binding by DrmAB}

The DNA-bound DrmAB complex includes a 7-mer ssDNA segment (Fig. 2a). While this is only a fragment of the DNA used for complex assembly, comprising a 19-bp duplex with a 5nucleotide hairpin on one end and a 7-nucleotide $5^{\prime}$ tail and a 21-nucleotide $3^{\prime}$ tail on the other end, we attributed this to the flexibility of DNA that does not make direct contact with the complex.

DrmA has multiple interactions with the backbone of the DNA, mostly electrostatic contacts between positively charged side chains and DNA phosphate groups (Fig. 2b \& c). Such nonspecific electrostatic DrmAB:DNA contacts provide the molecular basis for the previously observed broad anti-phage targeting by DISARM $^{7}$. Since conventional RM systems require recognition of specific DNA sequence motifs for nuclease activity, they can be easily evaded through phage evolving escape mutations ${ }^{11,23}$. However, by lacking sequence preferences for DNA binding, DISARM can provide effective anti-phage defense in the absence of a particular restriction site.

To address the functional relevance of these interactions, we mutated various DNA-interacting residues within DrmA (K803, R1294, R659, R810) to alanine. All four mutants showed reduced anti-phage protection in vivo (Fig. 2d). Together, these data underscore the functional role of DrmA as the DNA-targeting arm of the complex and provide the mechanism of targeting a wide range of phage by DISARM. Overall, the non-specific interactions between DrmA and DNA provide a structural basis for broad phage targeting by DISARM.

\section{ATPase activity is critical for DISARM function}

We observed strong density corresponding to ADP within our DNA-bound (Fig. 2e) and DNAfree maps, both of which were at the interface between the RecA1 and RecA2 domains, the canonical ATP-binding site of SF2 helicase domains. While the RecA domains of DrmA suggest that it is a helicase, the impact of its helicase activity on anti-phage targeting is poorly understood. We mutated four ADP-interacting residues to test their impact on DISARM activity. All four mutants showed reduced DISARM activity against a broad range of phage in vivo. This confirmed that in addition to DNA binding, ATP hydrolysis by DrmA is essential for DISARM activity. This is similar to type I RM systems, which encode a DEAD-box helicase domain protein that drives DNA translocation upon recognition of an unmethylated restriction site $^{10,24}$. Akin to DrmAB, ATP-dependent DNA translocation is essential for type I RM system activity ${ }^{25}$. However, it is unlikely that these two antiphage systems share a common DNA degradation mechanism, since type I RM systems translocate and cleave dsDNA, while DISARM appears to bind exclusively ssDNA (Figure 3). 


\section{DrmA contains an unstructured trigger loop that partially occludes the DNA-binding site}

Two dominant, distinct populations ( 120,000 particles each) emerged during focused 3D classification; one of which had strong DNA density (DrmAB:ADP:DNA) and one lacking observable DNA density (DrmAB:ADP). Comparison of our two maps revealed the presence of a 55-residue loop in DrmA that is otherwise absent in the DNA-bound structure (Fig. 3a \& b). Due to the flexibility of this loop, we were unable to confidently assign sequence position. We modeled this region as poly-alanine. To further validate this model, we determined a structure of DrmAB in the absence of DNA and ADP at a resolution of $3.8 \AA$ (Supplementary Fig 4). Rigid-body docking of our higher-resolution DrmAB:ADP model revealed density consistent with the presence of this loop (Supplementary Fig 4). Thus, the presence of this loop is mutually exclusive with DNA binding.

After superposition of our two models, we were surprised to observe a severe steric clash between DNA and this unstructured loop (Fig. 3c) that occurs at the interface between the 5' DNA end and RecA2. We hypothesized that this region of DrmA acts as a trigger loop (TL) that activates DrmAB after being dislodged by target DNA. Given that the presence of TL and DNA are mutually exclusive, we hypothesized that in the absence of single-stranded DNA, this loop may partially occlude the DNA binding site, preventing DrmAB from loading onto nonphage (i.e. host) DNA. To study its relevance in the DISARM mechanism, we created a mutant lacking this loop $(\operatorname{DrmA}(\Delta \mathrm{loop}))$, removing residues 181-233. Since $\operatorname{DrmA}(\Delta \mathrm{loop})$ maintained its ability to co-purify with $\operatorname{DrmB}$, and the $\operatorname{DrmA}(\Delta$ loop $) B$ complex eluted at the expected volume according to size-exclusion chromatography (Supplementary Fig. 1a), heterodimer assembly does not depend on this region of DrmA. We performed native electrophoretic mobility shift assays to compare binding of $\operatorname{DrmAB}$ and $\operatorname{DrmA}(\Delta \mathrm{loop}) \mathrm{B}$ to fluorescently-labelled 75-nt single-stranded DNA (Fig. 3d,e). While DrmAB bound DNA at concentrations of $1 \mu \mathrm{M}$ or higher, the presence of smeared bands likely indicated dissociation of DNA from the complex. In contrast, DNA dissociation from $\operatorname{DrmA}(\Delta \mathrm{loop}) \mathrm{B}$ bound DNA was not observed. Furthermore, a second super-shift occurred at protein concentrations of $1 \mu \mathrm{M}$ or higher, likely corresponding to multiple copies of the complex binding to the DNA concurrently, as observed for other nucleoprotein complexes ${ }^{26,27}$.

Since TL limits DNA binding, we performed additional binding assays to determine how this loop affects DNA substrate preferences of DrmAB (Fig. 3f,g). DrmAB bound to ssDNA and DNA with a 5'-overhang (ovh) but did not bind to dsDNA or DNA with a 3'ovh. DrmA( $\triangle$ loop)B displayed no DNA substrate preference, binding to ssDNA, dsDNA, and DNA with both a 5'ovh and 3'ovh. Thus, the TL limits structure-specific DNA binding by partially occluding the DNAbinding surface of DrmAB.

While the methylation modules of the DISARM operon recognize specific sequences, DISARM targets a diverse range of phage DNA sequences ${ }^{7}$. We thus propose that TL functions as a specificity filter, preventing interactions with dsDNA and DNA containing 3'ovh. Since DNA would occupy a larger surface on DrmAB than TL, DNA with a 5'ovh would be an ideal substrate for efficiently competing with TL for loading onto the complex. This may function as a mechanism for conferring substrate specificity to DISARM and prevent interactions with bacterial chromosome and other self-DNA, which would likely result in deleterious autoimmune effects. By relying on DNA structural context rather than simply DNA sequence, DISARM appears to utilize an alternative mechanism for avoiding self-targeting to many other nucleic acid-based antiphage systems ${ }^{5,24}$. 


\section{Methylation sensing and DNA-mediated DrmAB activation}

We sought to visualize additional conformational changes within DrmAB associated with DNA loading and complex activation. Within the core DNA-binding site of DrmAB, continuous cryoEM density is observed between the DNA nucleobase at position $3\left(\mathrm{~T}_{(3)}\right)$ (Fig. 2c) and the side-chain of DrmA(V1296), likely corresponding to a van der Waals interaction (Fig. 4a). Since this residue is highly conserved (found in $>98 \%$ of top 5000 DrmA homologs) and is the sole interaction between DrmAB and a DNA base, we hypothesized that DrmA(V1296) may act as a sensor for DNA methylation status. Serratia DISARM operon contains both a 5cytosine DNA methyltransferase and an N6-adenine DNA methyltransferase gene which target $\mathrm{ACAC}(\mathrm{mA}) \mathrm{G}$ and $\mathrm{YMT}(\mathrm{mC}) \mathrm{CAKR}$ motifs, respectively ${ }^{28}$ (Fig 1a), which may constitutively methylate the host genome to allow distinguishing self (i.e. methylated) from non-self (i.e. unmethylated) phage DNA, akin to RM systems ${ }^{7,11}$.

Structural analysis of our model revealed that DrmA(V1296) is in close contact with the faces of the DNA nucleobases, tightly wedged between DNA positions 3 and 4 . Modeling this position as a bulky tryptophan $(\mathrm{Y})$ side-chain showed severe steric clashes, and this mutant rendered DISARM unable to protect against phage in our in vivo assay (Fig 4b). We then tested the in vivo antiphage activity of DISARM with a glycine at this position (DrmA(V1296G)), which would no longer contact DNA nucleobases. This also resulted in severely reduced DISARM activity, supporting the notion that V1296 plays a critical role in DNA recognition (Fig 4b). We propose that since this interaction is critical to DISARM function, differences in DNA methylation status may perturb this interaction, providing a structural mechanism for enabling DISARM to differentiate between self- and non-self DNA and preventing autoimmune targeting.

To further test this hypothesis, we performed ATPase assay of DrmAB in the presence and absence of methylated and unmethylated DNAs. In the absence of DNA, DrmAB did not exhibit ATPase activity (Fig 4b). This is expected for a SF2 helicase, where DNA translocation is typically coupled to ATP hydrolysis ${ }^{29}$. ATPase activity is strongly stimulated by unmethylated DNA, but the ATPase rate is reduced in the presence of a DNA substrate containing three separate 5-methyl-cytosine bases (i.e. the modification provided by DrmMII7). This indicates that DrmAB not only discriminates between DNA overhangs (Fig 3) but also between methylated and unmethylated DNA substrates, which may serve as a second checkpoint for self- versus non-self discrimination.

We then compared our DNA-bound and DNA-free DrmAB complexes by superposing our models and generating motion vectors (Fig 1a). Upon loading of unmethylated DNA, we observed minor (mostly $<5 \AA$ ) conformational changes of the RecA1 and RecA2 domains (Fig. 4a). Surprisingly, we observed large conformational rearrangements in the $\mathrm{N}$-terminal half (NTH) of DrmB, despite a lack of any contacts with DNA. DrmB NTH shifts 10 A towards the RecA2 domain of DrmA, tightly clamping the complex around DNA.

This long-range allosteric communication appears to be mediated by the C-terminal region (CTR) of DrmA, which tightly wraps around the $\mathrm{N}$-terminus of DrmB acting as a pivot arm (PA). While DrmA(V1296) undergoes a minor shift upon recognition of unmethylated DNA, this is propagated into a much larger conformation change in the end of the PA and DrmB NTH. Thus, conformational change upon loading unmethylated DNA activates DrmAB.

DrmA PA is tightly interwoven with DrmB NTH, forming a myriad of contacts. These include a network of electrostatic interactions, hydrophobic contacts and $\pi-\pi$ stacking (Fig. 4d). We also observed that a five-residue segment of DrmA PA contributes to a 5 -stranded $\beta$-sheet in trans with $\mathrm{DrmB}$, forming an oligonucleotide/oligosaccharide-binding (OB) fold (Fig. 4e). The $\beta$ strand from DrmA is sandwiched between strands from DrmB, further locking the PA of DrmA in place. These intimate contacts allow DrmA to allosterically communicate with DrmB, setting in motion major conformational changes upon loading of unmethylated DNA. 
To test the importance of this conformational change for DISARM activity, we truncated DrmA so that it lacked the PA and tested the ability of DISARM to prevent phage replication in vivo. We found that DrmA- $\triangle$ PA had severely reduced DISARM activity against a broad range of phage, supporting the notion that the CTR-driven conformational changes of DrmAB result in DISARM activation (Fig. 4f). Based on our structural and functional data, we propose that upon displacement of the TL by ssDNA, DrmA(V1296) senses the DNA methylation status, allosterically activating DrmAB and triggering the DISARM antiphage response.

In summary, through coupling ATPase activity to DNA nucleobase methylation status, DrmAB may be able to achieve specific targeting of unmodified non-self (i.e. phage) DNA without relying on a given DNA sequence.

\section{Discussion}

We propose a model whereby DrmA and DrmB are expressed by the host cell in an autoinhibited form in the absence of phage infection. Through the constitutive expression of the core DrmAB complex, a DISARM response can be rapidly activated upon recognition of phage stimulus without necessitating transcription of either component, in accordance with previous observations ${ }^{7}$. In the absence of DNA, the DNA-binding channel of DrmAB is occluded by an unstructured $\sim 50$ residue loop within TL. While truncation of this loop does not affect heterodimer formation, we observed that $\operatorname{DrmA}(\Delta l o o p) B$ no longer exhibited discrimination for 5'ovh-containg DNA substrates and could bind both 3'ovh and dsDNA. We conclude that this loop may act as a selectivity filter or TL, ensuring the loading of specific substrates onto DrmAB. Since TL only partially occludes the DNA binding site, loading of 5'ovh DNA can effectively compete against and evict $T L$, which is likely intrinsically disordered. A similar mechanism of autoinhibition was recently reported for human Separase ${ }^{30}$, and examples of substrate-alleviated autoinhibition are numerous amongst other SF2 helicase complexes $^{31-34}$. Interestingly, a recent study of the human DEAD-box helicase DHX37 revealed a highly similar autoinhibition mechanism, whereby an unstructured protein loop occupied the substrate-binding channel${ }^{35}$. It may emerge that similar mechanisms of autoinhibition are widespread amongst a diverse group of SF2 helicase proteins from all domains of life.

Based on our data, we propose that the activating substrate for DISARM is unmodified 5'ovh DNA. Unlike many RM systems, DISARM does appear to not rely on specific sequences, enabling broad anti-phage targeting that cannot be circumvented escape mutants. The preference for 5'ovh ssDNA is particularly important since many dsDNA phage inject their genome in a linearized form with two sticky end 5'ovh, which subsequently become ligated during DNA circularization ${ }^{36,37}$, thus providing a stimulus for DISARM activation. This is supported by the previous observation that DISARM has no effect on phage adsorption but blocks phage DNA circularization?. If a dsDNA phage does not have such 5' ovh, then it would still likely be targeted by DISARM since it would still replicate via rolling circle DNA replication, where the leading strand is replicated in a 5' to 3' direction and the lagging strand is synthesized as Okazaki fragments ${ }^{38}$. This model explains the previous observation that DISARM is activated by the onset of phage replication, likely resulting probably in the degradation of viral DNA ${ }^{7}$. This data also explains how DrmAB target specificity is conferred by DNA structure rather than a specific sequence, providing an elegant mechanism for balancing broad DNA targeting with minimal autoimmune consequences. DISARM therefore represents a novel paradigm in bacterial antiviral defense mechanisms that target nucleic acids. Rather than achieving specific and efficient degradation of phage DNA through recognition of specific sequences, DISARM recognizes the structural context of DNA. The core DISARM complex DrmAB is able to confer specificity of DNA loading by targeting substrates with a $5^{\prime}$ ovh, thereby avoiding degradation of the host chromosomal DNA. This 
specificity is further conveyed by a sensitivity to DNA methylation status, whereby methylated DNA limits the ATPase activity of DrmAB which is essential for antiphage defense. This amounts to a two-pronged mechanism of selectivity that does not rely on a given DNA sequence, and thereby circumvents evasion via mutational escape.

Once activated, DrmAB binds to, translocates, and unwinds phage DNA, blocking replication and transcription and disrupting the phage infection cycle. Additionally, DrmAB may recruit a nuclease to trigger DNA degradation. Since the PLD-containing candidate nuclease DrmC is dispensable for DISARM activity and is often missing from annotated DISARM operons, it has remained a mystery how this is achieved ${ }^{7}$. However, since PLD-containing nucleases are highly abundant in bacterial genomes, we propose that DrmAB may recruit an alternative PLDnuclease protein. While previous in vivo assays were performed in Bacillus subtilis (which encodes a $42.2 \%$ identity DrmC-like homolog), our in vivo assays were performed in Escherichia coli (which encodes a 93.4\% identity DrmC-like homolog). The ubiquity and versatility of such DrmC-like non-specific PLD nucleases ${ }^{39-42}$ may make DrmC redundant, allowing DISARM to 'mix-and-match' components from other innate immune systems encoded by the host bacteria in order to achieve rapid and broad anti-phage protection.

Phage therapy represents a promising avenue to treat bacterial infection in an age where antibiotic resistance is widespread ${ }^{43}$. Since DISARM is an incredibly widespread mechanism of antiviral defense in bacteria, once we understand the fundamental mechanisms underlying DISARM activation it may be possible to develop small molecule treatments to inhibit DISARM, which could be delivered in conjunction with phage therapy to increase the effectiveness of such antibacterial treatment. 


\section{Methods}

\section{Cloning and protein expression}

Genes drmA and drmB were amplified from genomic DNA of Serratia sp. SCBI using primers $X$ and X (Sup. Table X) with Q5 DNA Polymerase (NEB: M0491S) as indicated by the manufacturer. Amplified gene drmB was cloned in plasmid 13S-S (Addgene: 48329), resulting in a $\mathrm{N}$-terminal $6 \mathrm{X}$ His tagged to the protein product. His-tag was separated from the gene with a tobacco etch virus (TEV) protease recognition sequence. Amplified gene drmA was cloned in plasmid pACYCDuet-1 (Sigma-Aldrich: 71147), resulting in a non-tagged protein product. Cloning products were transformed in Dh5 $\alpha$ using the heat-shock protocol ${ }^{46}$ and confirmed by sanger sequencing (Macrogen).

Both plasmids were transformed into E. coli BL21-AITM (Thermo Fisher) to express the DrmAB complex. Briefly, cultures were grown in LB medium at $37^{\circ} \mathrm{C}$ with shaking until exponential growth phase $\left(\mathrm{OD}_{600}\right.$ of 0.5$)$. After cooling down in ice for $20 \mathrm{~min}$, they were induced by addition of $0.2 \%(\mathrm{w} / \mathrm{v})$ L-arabinose and $1 \mathrm{mM}$ IPTG. After overnight incubation at $20^{\circ} \mathrm{C}$ with shaking, cells were pelleted by centrifugation, resuspended in Buffer A ( $25 \mathrm{mM} \mathrm{HEPES,} \mathrm{pH} 8$, $600 \mathrm{mM} \mathrm{NaCl}, 5 \%$ glycerol, $25 \mathrm{mM}$ imidazole, cOmplete ${ }^{\mathrm{TM}}$ EDTA-free Protease Inhibitor), and sonicated (10 cycles of $30 \mathrm{sec}$ separated by $30 \mathrm{sec}$ breaks, $40 \%$ amplitude). Cell lysate was pelleted down $\left(20,000 \mathrm{xg}, 60 \mathrm{~min}, 4^{\circ} \mathrm{C}\right)$ to remove cell debris, and supernatant was filtered with a $0.45 \mu \mathrm{m}$ PES filter and placed on ice until proceeding with the purification.

All the mutants used here were generated by round-the-horn side-directed mutagenesis and purified as the wild-type (WT) proteins.

\section{Protein purification}

Purification was performed at $4^{\circ} \mathrm{C}$ using an ÄKTA ${ }^{\mathrm{TM}}$ pure (GE Healthcare) to control flow and column pressure. Lysates were loaded onto a $5 \mathrm{~mL}$ Ni-NTA Superflow Cartridges (Qiagen) equilibrated with Buffer B (25 mM HEPES, pH 8, $600 \mathrm{mM} \mathrm{NaCl}, 5 \%$ glycerol, $25 \mathrm{mM}$ imidazole, $1 \mathrm{mM}$ DTT). Unbound lysate components were washed with 10 column volumes (CV) of Buffer B. Bound proteins were eluted with $5 \mathrm{CV}$ of Buffer $\mathrm{C}(25 \mathrm{mM}$ HEPES, $\mathrm{pH} 8,600 \mathrm{mM} \mathrm{NaCl}$, $5 \%$ glycerol, $150 \mathrm{mM}$ imidazole, $1 \mathrm{mM}$ DTT) and collected in fractions. After checking by SDSPAGE electrophoresis using a 4-20\% Mini-PROTEAN ${ }^{\circledR}$ TGX precast gel (Bio-Rad), fractions containing DrmAB were pooled together and buffer exchanged to Buffer D (25 mM HEPES, $\mathrm{pH} 8,150 \mathrm{mM} \mathrm{NaCl}, 5 \%$ glycerol, $1 \mathrm{mM}$ DTT). To separate the DrmAB complex from proteins that also bound the Ni-NTA column, sample was loaded onto a HiLoad 16/600 Superdex 200 (GE Healthcare). Column was washed with $2 \mathrm{CV}$ of Buffer D and fractions collected. After SDS-PAGE electrophoresis check, fractions containing the complex DrmAB were pooled together and concentrated using $30 \mathrm{KDa}$ NMWL Ultra-15 Amicon® (Merck). Protein concentration was estimated by the Bradford Assay (Thermo Scientific: 23246) as indicated by the manufacturer.

\section{Bacterial strains used in phage assays}

DISARM system genes from Serratia sp. SCBI were cloned in plasmids (Sup. Table XX), for both WT and mutant. Plasmids were transformed in E. coli BL21-Al. For assays, strains were cultured in LB media at $37^{\circ} \mathrm{C}$, and induced with $0.1 \%(\mathrm{w} / \mathrm{v}) \mathrm{L}$-arabinose and $0.5 \mathrm{mM} \mathrm{IPTG}$ at early exponential growth phase $\left(\mathrm{OD}_{600}\right.$ of $\left.0.2-0.3\right)$. Cultures were incubated at $37^{\circ} \mathrm{C}$ for 90 min and then used for phage assays. In parallel, a control strain (BL21-AI WT) was grown for all assays. When required, LB media was supplemented with antibiotics at the following final concentrations: $100 \mu \mathrm{g} / \mathrm{mL}$ ampicillin, $50 \mu \mathrm{g} / \mathrm{mL}$ kanamycin, $50 \mu \mathrm{g} / \mathrm{mL}$ streptomycin, $50 \mu \mathrm{g} / \mathrm{mL}$ spectinomycin, $10 \mu \mathrm{g} / \mathrm{mL}$ chloramphenicol. 


\section{Phage strains}

E. coli phages T1, T7, and Nami were used in this study. For their production, phages T1 and T7 were propagated in E. coli BL21-AI, as described previously ${ }^{47}$, and bacteriophage Nami in its host E. coli isolate R10256 following same procedure. Briefly, bacterial cultures at exponential growth phase (aprox. $0.4 \mathrm{OD}_{600}$ in a $10 \mathrm{~mm}$ cuvette readers) were infected with a phage lysate and incubated overnight. Then, cultures were spun down and the supernatant filtered through $0.2 \mu \mathrm{m}$ PES membranes. When required, phages were concentrated by addition of PEG-8000 and $\mathrm{NaCl}$ at final concentrations of $100 \mathrm{mg} / \mathrm{mL}$ and $1 \mathrm{M}$, respectively. Following, it was incubated overnight at $4^{\circ} \mathrm{C}$, centrifuged at $11,000 \mathrm{xg}$ at $4^{\circ} \mathrm{C}$ for $60 \mathrm{~min}$, and the phage-containing pellet resuspended in the desired final volume of Saline Magnesium (SM) buffer. Phage stocks were stored at $4^{\circ} \mathrm{C}$ before their use and titer determined as indicated below.

\section{Phage titering}

Bacteria cultures in exponential growth phase were used to titer phages stocks. For this, 100 $\mu \mathrm{L}$ of culture were mixed with $5 \mathrm{~mL}$ of $0.6 \% \mathrm{LBA}$ at $45^{\circ} \mathrm{C}$ and poured to a LBA plate to form a bacterial layer. Ten-fold dilution of phages in SM buffer or LB media were plated on the top of the bacteria in $10 \mu \mathrm{L}$ drops and let dry for $20 \mathrm{~min}$. Plates were incubated overnight up-side down at $37^{\circ} \mathrm{C}$. To determine the phage titer, the number of center of infections (plaques) were counted. E. coli BL21-Al was used for titering phages T1, T7, and Nami.

\section{Efficiency of plating determination}

To determine the efficiency of plating (EOP), phages were plated on the induced strains containing the DISARM system and compared to the plating on control strain. For this, $200 \mu \mathrm{L}$ of the bacterial cultures were mixed a specific, countable number of infectious particles (50$150 \mathrm{PFU} /$ plate) and $4.5 \mathrm{~mL}$ of $0.6 \% \mathrm{LBA}$ at prewarmed at $45^{\circ} \mathrm{C}$. The mixture was then poured on top of a LBA plate to form a bacterial layer containing the infectious particles. After overnight incubation at $37^{\circ} \mathrm{C}$, the EOP was calculated by dividing the number of plaques counted on each plate by the number of plaques formed in the control strain.

\section{CryoEM sample preparation, data collection and processing}

To capture DrmAB in the act of unwinding a DNA substrate, a forked DNA construct consisting of a 19-bp stem-loop structure with a 6 base 5' overhang and a 20 base 3' overhang was designed. This substrate was chosen because similar substrates have been used to capture unwinding intermediates of bacterial SF2 helicase complexes ${ }^{48,49}$. DrmAB was buffer exchanged into cryoEM sample buffer $(150 \mathrm{mM} \mathrm{NaCl}, 25 \mathrm{mM}$ HEPES pH 7.4) through repeated application of buffer to sample within a $0.5 \mathrm{ml}$ spin concentrator, with a $30 \mathrm{kDa}$ size cutoff. $10 \mu \mathrm{M}$ DrmAB was incubated with 10-fold excess DNA stem loop (100 $\mu \mathrm{M})$ and 100fold excess ADP (1 mM). Complex formation was monitored using electrophoretic mobility shift assay (EMSA), confirming DNA binding by DrmAB (Supplementary Fig 1). After incubation at room temperature $\left(\sim 25^{\circ} \mathrm{C}\right)$ for 30 minutes, DrmAB:ADP:DNA was applied to glow discharged holey carbon grids (C-flat 2/2, Protochips Inc.), blotted for $2 \mathrm{~s}$ with a blot force of 4 and rapidly plunged into liquid ethane using an FEI Vitrobot MarkIV.

Data was collected on an FEI Titan Krios cryo-electron microscope equipped with a K3 Summit direct electron detector (Gatan, Pleasanton, CA). Images were recorded with SerialEM ${ }^{50}$ with a pixel size of $1.1 \AA$ over a defocus range of -1.5 to $-2.5 \mu \mathrm{m}$. During early stages of data collection, a preferred orientation was observed. To ameliorate this, a dataset of 6828 micrographs was collected at $30^{\circ}$ tilt, in addition to the original 2548 micrographs collected without tilt. Movies were recorded at 13.3 electrons/pixel/second for 6 seconds ( 80 frames) to give a total dose of 80 electrons/pixel. CTF correction, motion correction and particle picking were performed in real-time using WARP51, resulting in 4,669,932 particles, which were uploaded to cryoSPARC v2 ${ }^{52}$ (Supplementary Fig 2). 
Multiple rounds of 3D classification within cryoSPARC yielded a final set of 144,989 particles that gave a 3D reconstruction at a global resolution of $2.84 \AA$ using non-uniform refinement ${ }^{53}$. However, since bound DNA had weak density, an alternative data processing strategy was implemented. Two rounds of ab initio reconstruction followed by heterogeneous refinement were performed on the $0^{\circ}$ and $30^{\circ}$ tilt datasets separately within cryoSPARC. The resulting subset of undamaged particles was combined and yielded a $3.1 \AA$ reconstruction. These particles were then imported into Relion $3.1^{54}$, where masks covering the core of the complex (to improve DNA density) and the bottom of the complex (to improve the quality of DrmB density) were generated within ChimeraX ${ }^{55}$ and Relion. These masks were then used for focused 3D classification within Relion $(N=6, T=25)$. Particles within classes corresponding to DrmAB:ADP:DNA and DrmAB:ADP were then selected for Relion 3D auto-refinement and post-processing, resulting in structures with global resolutions of $3.4 \AA$ and $3.3 \AA$, respectively (Supplementary Fig 2).

To assist de novo model building, the structures of multiple overlapping regions of DrmA and DrmB were predicted using trRosetta ${ }^{56}$. These models were initially fitted into the map as rigid bodies using ChimeraX. Once suitable fits were found, bulk flexible fitting was performed using Isolde and Namdinator ${ }^{57}$. Real-space model improvement was then performed using Isolde ${ }^{58}$, and final models were subjected to real-space refinement in Phenix ${ }^{59}$. Structural figures were prepared using ChimeraX. Structural analysis of sequence conservation was performed using ConSurf ${ }^{60}$, and visualized in ChimeraX. Motion vectors to visualize the conformational change from DrmAB:ADP to DrmAB:ADP:DNA were generated and visualized using PyMol.

\section{Native electrophoretic mobility shift assays (EMSAs)}

To determine the DNA substrate preferences of DrmAB, 5'Cy5-labelled 75-nt DNA was incubated with 1.5-fold excess complement, 5 'block or 3 'block oligos (Table 2) and heat annealed at $85^{\circ} \mathrm{C}$ for 10 minutes prior to cooling to $4^{\circ} \mathrm{C} .5 \mathrm{nM}$ DNA was incubated with $1 \mu \mathrm{M}$ $\operatorname{DrmAB}$ or $\operatorname{DrmA}(\triangle \mathrm{loop}) \mathrm{B}$ in cryoEM sample buffer for 30 minutes at room temperature in a total volume of $20 \mu \mathrm{l}$. A total of $10 \mu \mathrm{l}$ of each sample was mixed with $2 \mu \mathrm{L} 6 \times$ loading buffer (30\% v/v glycerol, 5\% Ficoll 400, $50 \mathrm{mM} \mathrm{NaCl}, 10 \mathrm{mM}$ HEPES pH 8, $5 \mathrm{mM}$ EDTA, 0.002\% $\mathrm{w} / \mathrm{v}$ bromophenol blue). Electrophoresis was carried out on a non-denaturing $1.5 \%$ agarose gel for $90 \mathrm{~min}$ at $100 \mathrm{~V}$ in $1 \times$ Tris-borate-EDTA buffer. Gels were imaged using a fluorescence scanner (Fujifilm FLA-5100) with 532 nm excitation.

For comparison of DNA binding between $\operatorname{DrmAB}$ and $\operatorname{DrmA}(\Delta \mathrm{loop}) \mathrm{B}$, serial 2-fold dilutions from of either protein complex were incubated with $5 \mathrm{nM}$ Cy5-labelled DNA. Samples were incubated and EMSAs were performed as described above.

\section{Phage strains}

E. coli phage T1, T7, and Nami were used in this study. For their production, phage T1 and T7 were propagated in E. coli BL21-AI, as described previously ${ }^{47}$, and bacteriophage Nami in its host $E$. coli isolate R10256 following same procedure. Briefly, bacterial cultures at exponential growth phase (aprox. $0.4 \mathrm{OD}_{600}$ in a $10 \mathrm{~mm}$ cuvette readers) were infected with a phage lysate and incubated overnight. Then, cultures were spun down and the supernatant filtered through $0.2 \mu \mathrm{m}$ PES membranes. When required, phage were concentrated by addition of PEG-8000 and $\mathrm{NaCl}$ at final concentrations of $100 \mathrm{mg} / \mathrm{mL}$ and $1 \mathrm{M}$, respectively. Following, it was incubated overnight at $4^{\circ} \mathrm{C}$, centrifuged at $11,000 \mathrm{xg}$ at $4^{\circ} \mathrm{C}$ for $60 \mathrm{~min}$, and the phage-containing pellet resuspended in the desired final volume of Saline Magnesium (SM) buffer. Phage stocks were stored at $4^{\circ} \mathrm{C}$ before their use and titer determined as indicated below.

\section{Phage titering}

Bacteria cultures in exponential growth phase were used to titer phage stocks. For this, 100 $\mu \mathrm{L}$ of culture were mixed with $5 \mathrm{~mL}$ of $0.6 \% \mathrm{LBA}$ at $45^{\circ} \mathrm{C}$ and poured to a LBA plate to form 
a bacterial layer. Ten-fold dilution of phage in SM buffer or LB media were plated on the top of the bacteria in $10 \mu \mathrm{L}$ drops and let dry for $20 \mathrm{~min}$. Plates were incubated overnight up-side down at $37^{\circ} \mathrm{C}$. To determine the phage titer, the number of center of infections (plaques) were counted. E. coli BL21-Al was used for titering phage T1, T7, and Nami.

\section{Efficiency of plating determination}

To determine the efficiency of plating (EOP), phage were plated on the induced containing the DISARM system and compared to the plating on control strain BL21AI. For this, $200 \mu \mathrm{L}$ of the bacterial cultures were mixed a specific, countable number of infectious particles (50-150 PFU/plate) and $4.5 \mathrm{~mL}$ of $6 \%$ LBA at $45^{\circ} \mathrm{C}$. The mixture was then poured on top of a LBA plate to form a bacterial layer containing the infectious particles. After overnight incubation at $37^{\circ} \mathrm{C}$, the EOP was calculated by dividing the number of plaques counted on each plate by the number of plaques formed in the control strain. 


\section{References}

1. Hampton, H. G., Watson, B. N. J. \& Fineran, P. C. The arms race between bacteria and their phage foes. Nature 577, 327-336 (2020).

2. $\quad$ Egido, J. E., Costa, A. R., Aparicio-maldonado, C., Haas, P. \& Brouns, S. J. J. Mechanisms and clinical importance of bacteriophage resistance. FEMS Microbiol. Rev. 1-16 (2021).

3. Labrie, S. J., Samson, J. E. \& Moineau, S. Bacteriophage resistance mechanisms. Nat. Rev. Microbiol. 8, 317-327 (2010).

4. Samson, J. E., Magadán, A. H., Sabri, M. \& Moineau, S. Revenge of the phages: Defeating bacterial defences. Nat. Rev. Microbiol. 11, 675-687 (2013).

5. Hille, F. et al. The Biology of CRISPR-Cas: Backward and Forward. Cell 172, 12391259 (2018).

6. Bravo, J. P. K., Liu, M., Mccool, R. S. \& Jung, K. Structural basis for mismatch surveillance by CRISPR / Cas9. bioRxiv 1-22 (2021).

7. Ofir, G. et al. DISARM is a widespread bacterial defence system with broad antiphage activities. Nat. Microbiol. 3, 90-98 (2017).

8. Doron, S. et al. Systematic discovery of antiphage defense systems in the microbial pangenome. Science (80-. ). 359, 0-12 (2018).

9. Xiong, X. et al. SspABCD-SspE is a phosphorothioation-sensing bacterial defence system with broad anti-phage activities. Nat. Microbiol. 5, 917-928 (2020).

10. Gao, Y. et al. Structural insights into assembly, operation and inhibition of a type I restriction-modification system. Nat. Microbiol. 5, 1107-1118 (2020).

11. Rusinov, I. S., Ershova, A. S., Karyagina, A. S., Spirin, S. A. \& Alexeevski, A. V. Avoidance of recognition sites of restriction-modification systems is a widespread but not universal anti-restriction strategy of prokaryotic viruses. BMC Genomics 19, 1-11 (2018).

12. Thiaville, J. J. et al. Novel genomic island modifies DNA with 7-deazaguanine derivatives. Proc. Natl. Acad. Sci. U. S. A. 113, E1452-E1459 (2016).

13. Ofir, G. \& Sorek, R. Contemporary Phage Biology: From Classic Models to New Insights. Cell 172, 1260-1270 (2018).

14. Holm, L. Using Dali for Protein Structure Comparison. Methods Mol. Biol. 2112, 29-42 (2020).

15. Makarova, K. S., Wolf, Y. I., Snir, S. \& Koonin, E. V. Defense Islands in Bacterial and Archaeal Genomes and Prediction of Novel Defense Systems. J. Bacteriol. 193, 6039-6056 (2011).

16. Roske, J. J., Liu, S., Wahl, M. C., Loll, B. \& Neu, U. A skipping rope translocation mechanism in a widespread family of DNA repair helicases. Nucleic Acids Res. 1-15 (2020) doi:10.1093/nar/gkaa1174.

17. Yakovleva, L. \& Shuman, S. Mycobacterium smegmatis SftH exemplifies a distinctive clade of superfamily II DNA-dependent ATPases with 3' to 5' translocase and helicase activities. Nucleic Acids Res. 40, 7465-7475 (2012).

18. Pace, N. J. \& Weerapana, E. Zinc-binding cysteines: diverse functions and structural motifs. Biomolecules 4, 419-434 (2014).

19. Vázquez-Torres, A. Redox active thiol sensors of oxidative and nitrosative stress. Antioxidants Redox Signal. 17, 1201-1214 (2012).

20. Fang, F. C., Frawley, E. R., Tapscott, T. \& Vázquez-Torres, A. Discrimination and Integration of Stress Signals by Pathogenic Bacteria. Cell Host Microbe 20, 144-153 (2016).

21. Fang, F. C., Frawley, E. R., Tapscott, T. \& Vázquez-Torres, A. Bacterial Stress Responses during Host Infection. Cell Host Microbe 20, 133-143 (2016).

22. Smith, L. M. et al. The Rcs stress response inversely controls surface and CRISPRCas adaptive immunity to discriminate plasmids and phages. Nat. Microbiol. 6, 162- 
$172(2021)$.

23. Pleška, M. \& Guet, C. C. Effects of mutations in phage restriction sites during escape from restriction-modification. Biol. Lett. 13, 9-12 (2017).

24. Murray, N. E. Type I Restriction Systems: Sophisticated Molecular Machines (a Legacy of Bertani and Weigle). Microbiol. Mol. Biol. Rev. 64, 412-434 (2000).

25. Davies, G. P., Powell, L. M., Webb, J. L., Cooper, L. P. \& Murray, N. E. EcoKI with an amino acid substitution in any one of seven DEAD-box motifs has impaired ATPase and endonuclease activities. Nucleic Acids Res. 26, 4828-4836 (1998).

26. Bravo, J. P. K. et al. Stability of local secondary structure determines selectivity of viral RNA chaperones. Nucleic Acids Res. 293191 (2018) doi:10.1101/293191.

27. Bravo, J. P. K. et al. Structural basis of rotavirus RNA chaperone displacement and RNA annealing. Proc. Natl. Acad. Sci. 1-35 (2021) doi:10.1073/pnas.2100198118.

28. Aparicio-Maldonado, C. et al. DISARM systems exploit DNA methylation to distinguish non-self molecules and overcome a broad range of invaders. bioRxiv.

29. Fairman-Williams, M. E., Guenther, U. P. \& Jankowsky, E. SF1 and SF2 helicases: Family matters. Curr. Opin. Struct. Biol. 20, 313-324 (2010).

30. Yu, J. et al. Structural basis of human separase regulation by securin and CDK1cyclin B1. Nature 000, (2021).

31. Schmidt, C. et al. The cryo-EM structure of a ribosome-Ski2-Ski3-Ski8 helicase complex. Science (80-. ). 354, 1431-1433 (2016).

32. Richards, J. D. et al. Structure of the DNA repair helicase Hel308 reveals DNA binding and autoinhibitory domains. J. Biol. Chem. 283, 5118-5126 (2008).

33. Lohman, T. M., Tomko, E. J. \& Wu, C. G. Non-hexameric DNA helicases and translocases: Mechanisms and regulation. Nat. Rev. Mol. Cell Biol. 9, 391-401 (2008).

34. Sloan, K. E. \& Bohnsack, M. T. Unravelling the Mechanisms of RNA Helicase Regulation. Trends Biochem. Sci. 43, 237-250 (2018).

35. Singh, S., Vanden Broeck, A., Miller, L., Chaker-Margot, M. \& Klinge, S. Nucleolar maturation of the human small subunit processome. Science (80-. ). 373, (2021).

36. Weigel, C. \& Seitz, H. Bacteriophage replication modules. FEMS Microbiol. Rev. 30, 321-381 (2006).

37. Molineux, I. J. \& Panja, D. Popping the cork: Mechanisms of phage genome ejection. Nat. Rev. Microbiol. 11, 194-204 (2013).

38. Taylor, K. \& Weggrzyn, G. Replication of coliphage lambda DNA. FEMS Microbiol. Rev. 17, 109-119 (1995).

39. Yang, W. Nucleases: Diversity of structure, function and mechanism. Quarterly Reviews of Biophysics vol. 44 (2011).

40. Schwardmann, L. S., Nölle, V. \& Elleuche, S. Bacterial non-specific nucleases of the phospholipase D superfamily and their biotechnological potential. Appl. Microbiol. Biotechnol. 104, 3293-3304 (2020).

41. Sasnauskas, G. et al. A novel mechanism for the scission of double-stranded DNA: Bfil cuts both $3^{\prime}-5^{\prime}$ and 5'-3' strands by rotating a single active site. Nucleic Acids Res. 38, 2399-2410 (2010).

42. Grazulis, S. et al. Structure of the metal-independent restriction enzyme Bfil reveals fusion of a specific DNA-binding domain with a nonspecific nuclease. Proc. Natl. Acad. Sci. U. S. A. 102, 15797-15802 (2005).

43. Furfaro, L. L., Payne, M. S. \& Chang, B. J. Bacteriophage Therapy: Clinical Trials and Regulatory Hurdles. Front. Cell. Infect. Microbiol. 8, 376 (2018).

44. Zi Tan, Y. et al. Addressing preferred specimen orientation in single-particle cryoEMthrough tilting. Nat. Methods 14, 793-796 (2017).

45. Jumper, J. et al. Highly accurate protein structure prediction with AlphaFold. Nature (2021) doi:10.1038/s41586-021-03819-2. 
46. Kostylev, M., Otwell, A. E., Richardson, R. E. \& Suzuki, Y. Cloning should be simple: Escherichia coli DH5á-mediated assembly of multiple DNA fragments with short end homologies. PLoS One 10, 1-15 (2015).

47. Bonilla, N. et al. Phage on tap-a quick and efficient protocol for the preparation of bacteriophage laboratory stocks. PeerJ 4, e2261 (2016).

48. Jia, N. et al. Structures and single-molecule analysis of bacterial motor nuclease AdnAB illuminate the mechanism of DNA double-strand break resection. Proc. Natl. Acad. Sci. U. S. A. 116, 24507-24516 (2019).

49. Cheng, K., Wilkinson, M., Chaban, Y. \& Wigley, D. B. A conformational switch in response to Chi converts RecBCD from phage destruction to DNA repair. Nat. Struct. Mol. Biol. 27, 71-77 (2020).

50. Mastronarde, D. N. Automated electron microscope tomography using robust prediction of specimen movements. J. Struct. Biol. 152, 36-51 (2005).

51. Tegunov, D. \& Cramer, P. Real-time cryo-electron microscopy data preprocessing with Warp. Nat. Methods 16, 1146-1152 (2019).

52. Punjani, A., Rubinstein, J. L., Fleet, D. J. \& Brubaker, M. A. CryoSPARC: Algorithms for rapid unsupervised cryo-EM structure determination. Nat. Methods 14, 290-296 (2017).

53. Punjani, A., Zhang, H. \& Fleet, D. J. Non-uniform refinement: adaptive regularization improves single-particle cryo-EM reconstruction. Nat. Methods 17, 1214-1221 (2020).

54. Zivanov, J. et al. RELION-3: new tools for automated high-resolution cryo-EM structure determination. Elife 421123 (2018) doi:10.1101/421123.

55. Pettersen, E. F. et al. UCSF ChimeraX: Structure visualization for researchers, educators, and developers. Protein Sci. 30, 70-82 (2021).

56. Yang, J. et al. Improved protein structure prediction using predicted interresidue orientations. Proc. Natl. Acad. Sci. U. S. A. 117, 1496-1503 (2020).

57. Kidmose, R. T. et al. Namdinator - Automatic molecular dynamics flexible fitting of structural models into cryo-EM and crystallography experimental maps. IUCrJ 6, 526531 (2019).

58. Croll, T. I. ISOLDE: a physically realistic environment for model building into lowresolution electron-density maps. Acta Crystallogr. Sect. D 74, 519-530 (2018).

59. Afonine, P. V. et al. Real-space refinement in PHENIX for cryo-EM and crystallography. Acta Crystallogr. Sect. D Struct. Biol. 74, 531-544 (2018).

60. Ashkenazy, H. et al. ConSurf 2016: an improved methodology to estimate and visualize evolutionary conservation in macromolecules. Nucleic Acids Res. 44, W344W350 (2016). 


\section{Data and availability}

The cryo-EM structure and associated atomic model of DrmAB-ADP have been deposited into the Electron Microscopy Data Bank and the Protein Data Bank with accession codes EMD24938and PDB 7S9V, respectively. The cryo-EM structure of DrmAB-ADP-DNA and associated atomic model have been deposited with accession codes EMD-24939and PDB 7S9W, respectively.

\section{Acknowledgements}

We thank members of the Taylor and Brouns groups for comments and helpful discussions; J. Yelland in the Taylor group for assistance with the ATPase assay; and A.C. Haagsma in the Brouns group for help in protein purification. The model cartoon was created using Biorender. This work was supported in part by the Netherlands Organisation for Scientific Research (NWO VICl; VI.C.182.027 to S.J.J.B). National Institute of General Medical Sciences (NIGMS) of the National Institutes of Health (NIH) R35GM138348 (to D.W.T.). D.W.T is a CPRIT Scholar supported by the Cancer Prevention and Research Institute of Texas (RR160088) and an Army Young Investigator supported by the Army Research Office (W911NF-19-1-0021).

\section{Author Contributions}

C.A-M cloned, expressed and purified all protein samples, performed and analyzed in vivo EOP assays. J.P.K.B. performed cryo-EM, structure determination, and modeling, and performed in vitro assays. J.P.K.B., C.A-M., S.J.J.B. and D.W.T. analyzed and interpreted the data and wrote the manuscript. S.J.J.B. and D.W.T supervised and secured funding for the studies.

\section{Competing interests}

The authors declare no competing interests.

\section{Materials and Correspondence}

Materials and correspondence requests should be addressed to Stan J.J. Brouns (S.J.J.Brouns@tudelft.nl) and David W. Taylor (dtaylor@utexas.edu). 

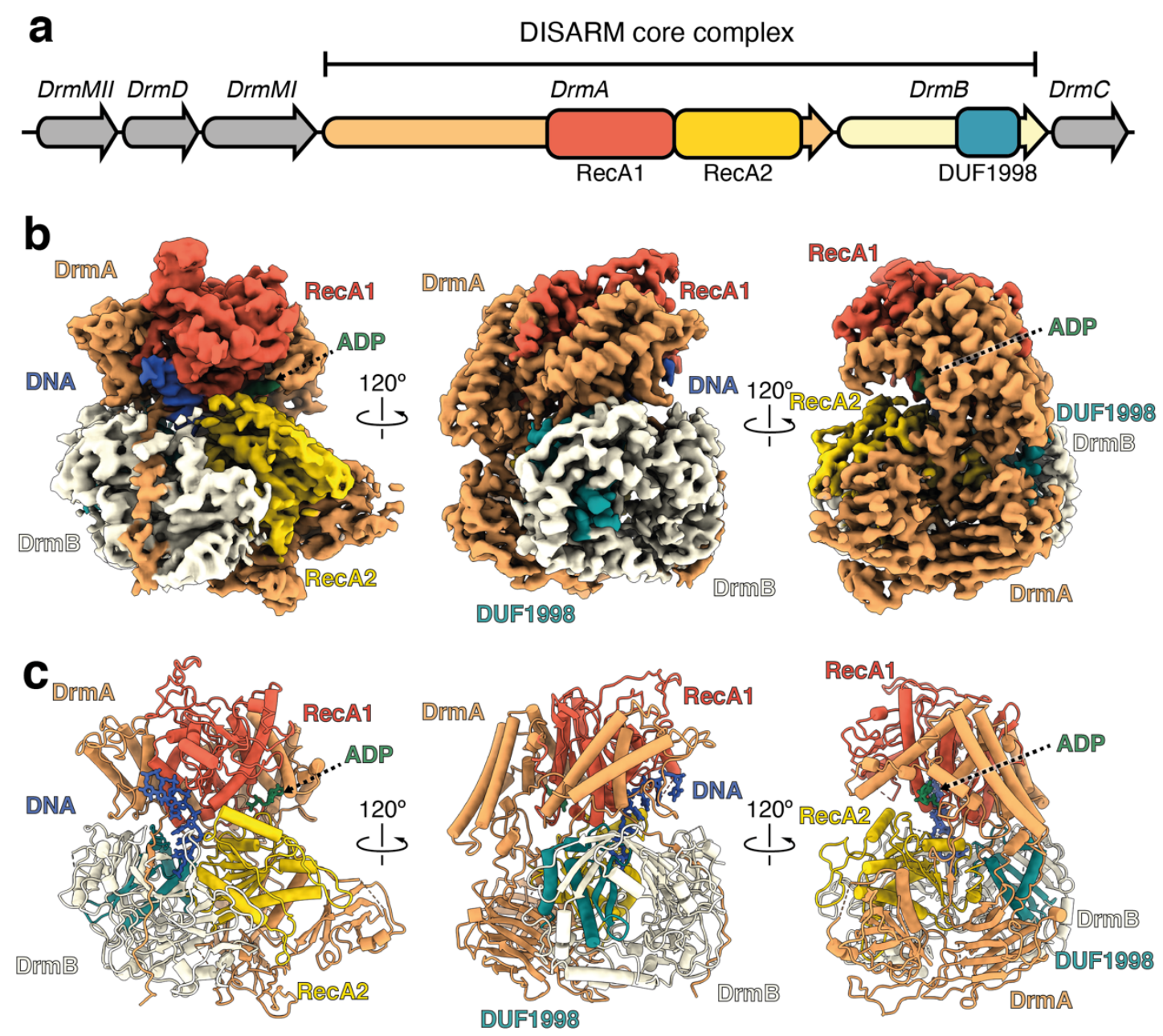

Fig. 1 I Architecture of the DrmAB:ADP:DNA complex. a, Serratia sp. DISARM operon. Conserved DISARM core components DrmA and DrmB are colored by structural domains. $\mathbf{b}$, Cryo-EM reconstruction of DrmAB:ADP:DNA complex colored as in a. ssDNA is shown in blue and ADP in green. $\mathbf{c}$, Atomic models built into the cryo-EM map (b). 

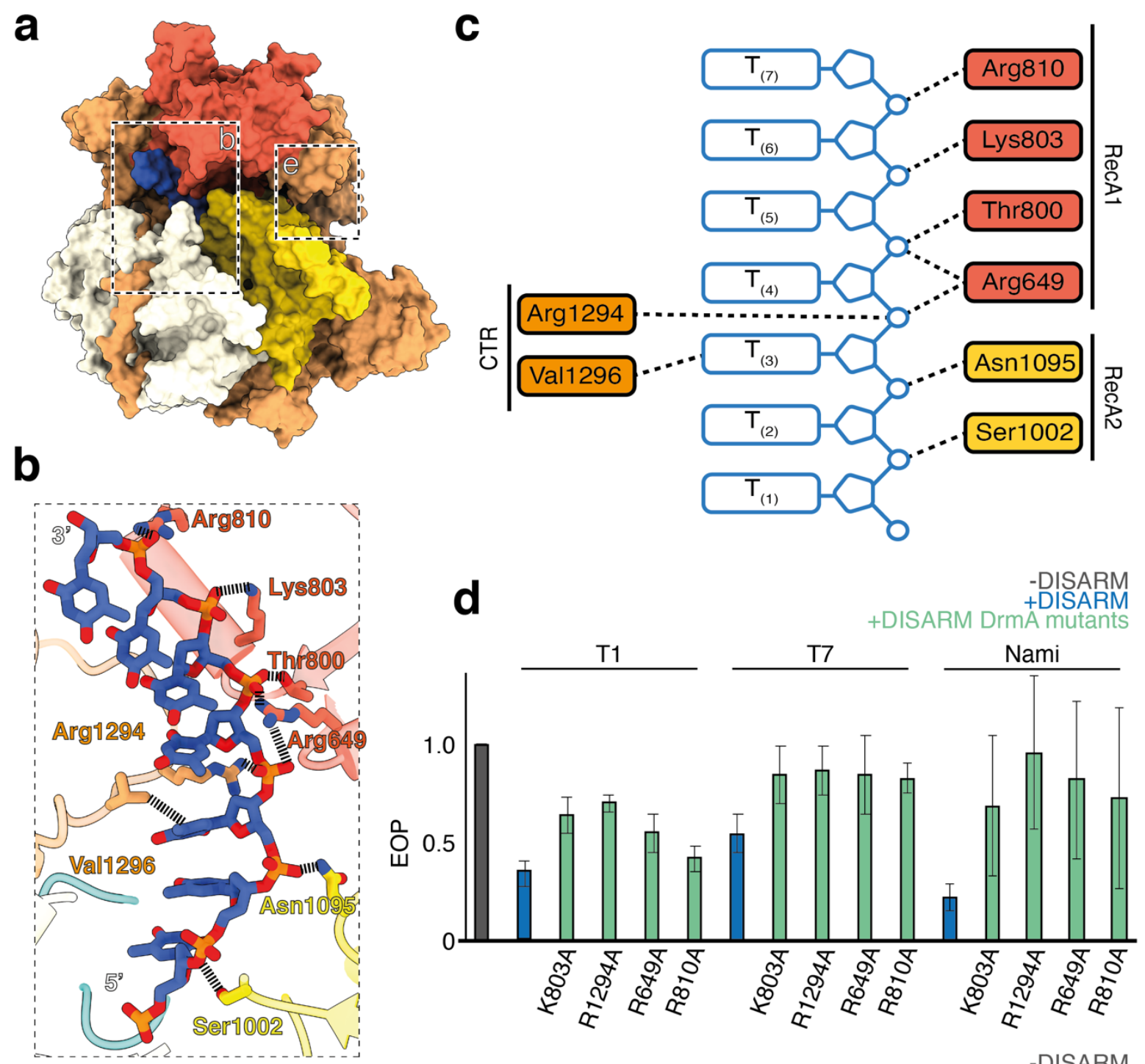

e
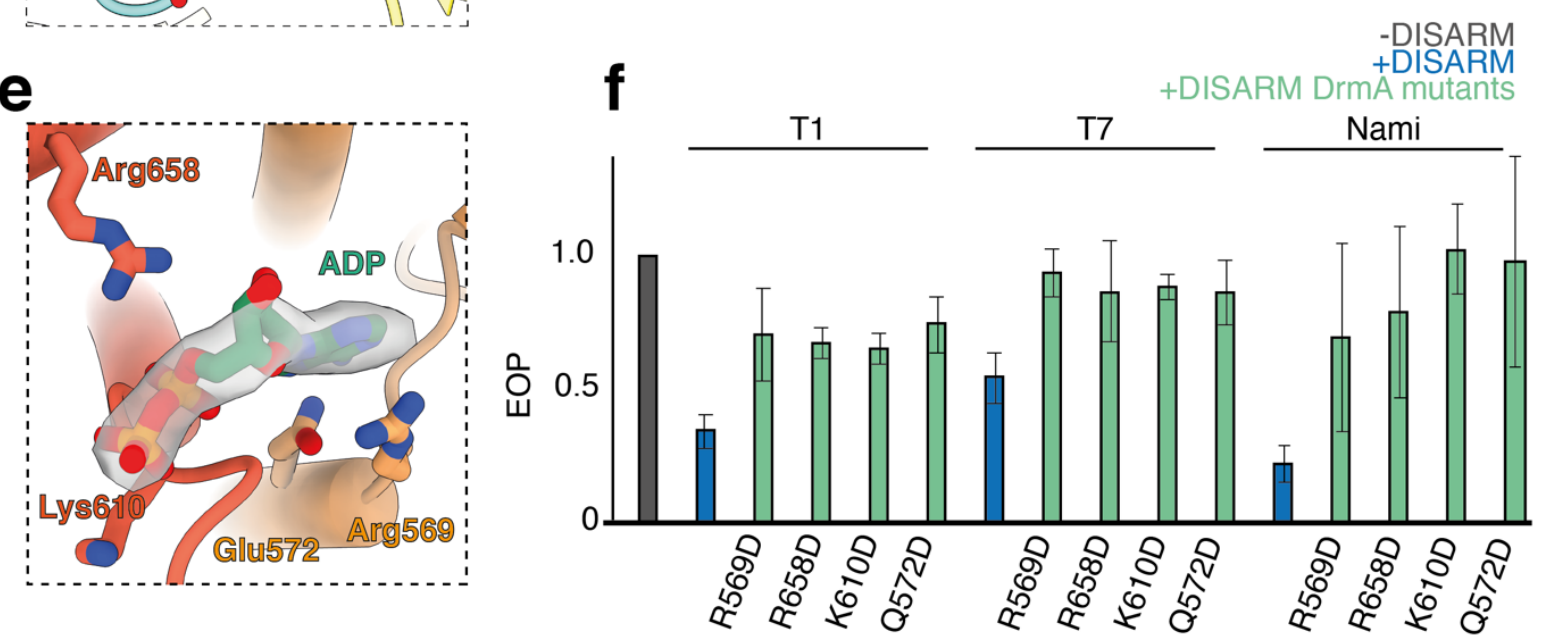

Fig. 2 I Both ssDNA and nucleotide cofactor binding are essential for DISARM function. a, Overall structure of DrmAB:DNA:ADP. Boxes indicate regions featured in panels $\mathbf{b}$ and $\mathbf{e}$. $\mathbf{b}$, Close-up view of DrmA:DNA interactions. No interactions between DrmB and DNA are observed. c, Schematic of DNA-protein contacts colored by protein domain. $\mathbf{d}$, Effect of mutagenesis of DrmA DNA-interacting residues on DISARM anti-phage activity. $\mathbf{e}$, Close-up view of DrmA:ADP interactions. $\mathbf{f}$, Effect of mutagenesis of DrmA ADP-interacting residues on DISARM anti-phage activity. 

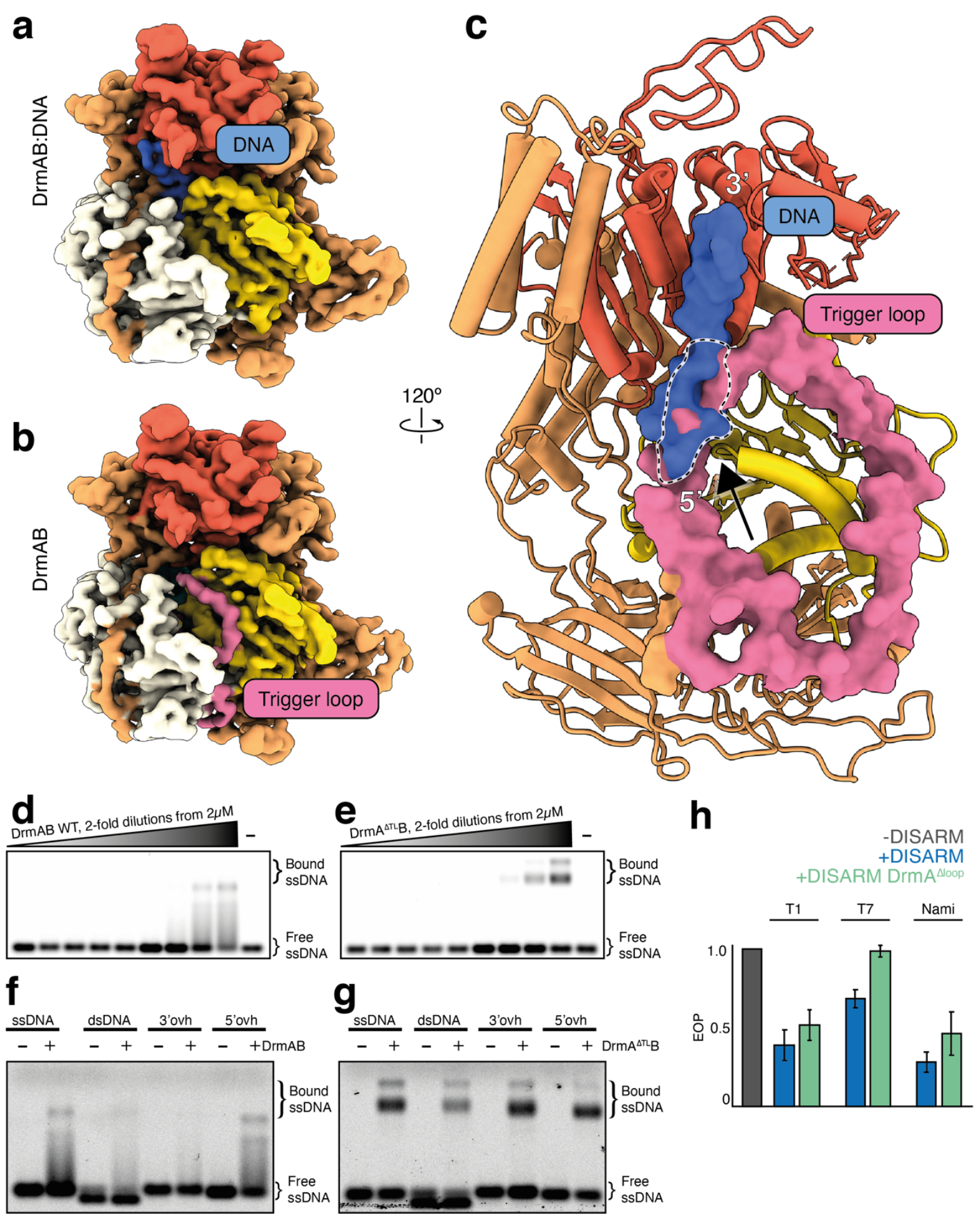

Fig. 3 I DrmA trigger loop (TL) partially occludes DNA-binding site. a \& b, Cryo-EM reconstruction of apo DrmAB (a) and DNA-bound DrmAB (b). Density corresponding to DrmA loop is shown in pink. c, Overlay of DrmAB-bound DNA (blue) and TL (pink) showing the steric clash (dashed black \& white outline). TL partially occludes DNA-binding site. d \& e, EMSA analysis of DrmAB and $\operatorname{DrmA}(\Delta \mathrm{loop}) \mathrm{B}$ binding DNA. The smeared bands that occur at high concentrations of DrmAB correspond to bound DNA dissociating from the complex. This did not occur for $\operatorname{DrmA}(\Delta \mathrm{loop}) \mathrm{B}$, and an additional super-shift was present, corresponding to multiple copies of DrmA( $\triangle$ loop)B binding to the same 75-nt DNA concurrently. f \& $\mathbf{g}$, DNA substrate preferences of $\operatorname{DrmAB}$ and $\operatorname{DrmA}(\Delta \mathrm{loop}) \mathrm{B}$. The 75-nt Cy5-labelled DNA (ssDNA) was annealed to complementary oligos corresponding to the full sequence (dsDNA), or 20 
bases at the 5' or 3' ends (3'ovh and 5'ovh, respectively). DrmAB showed a preference for ssDNA and 5'ovh DNA, whereas $\operatorname{DrmA}(\Delta$ loop)B bound to all DNA substrates tested. $\mathbf{h}$, Effect of truncation of TL on DISARM anti-phage activity. 

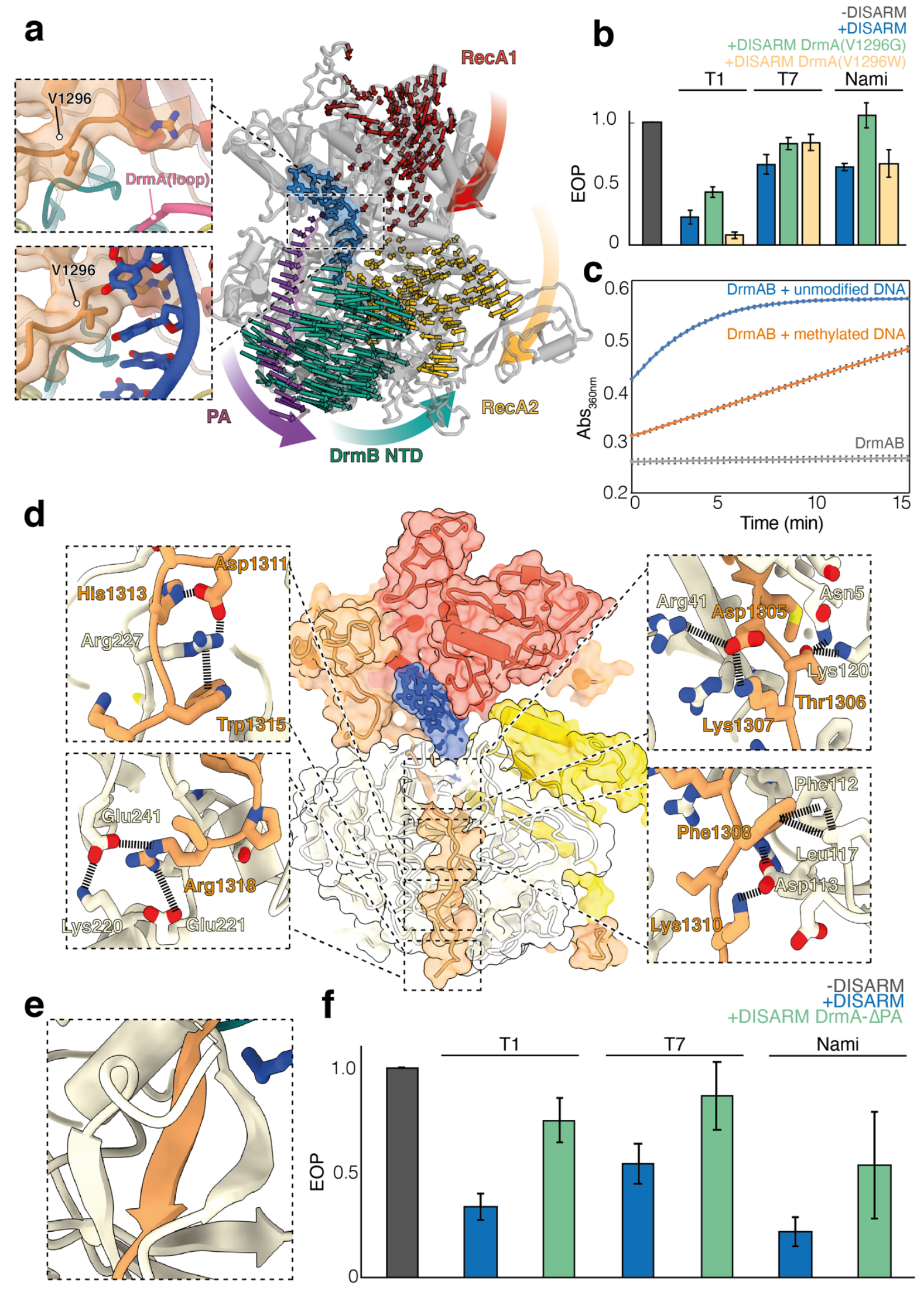

Fig. 4 I Binding of unmethylated ssDNA activates DrmAB through large conformational rearrangements. a, DrmA C-terminal pivot arm (PA) interface with substrate DNA. Left: closeup views of DrmAB map and model lacking DNA (top) and DrmAB:DNA map and model 
(bottom). In the DNA-bound map, a direct interaction between a DNA base and Val1296 is present (black arrow). In the DrmAB map lacking DNA, the DrmA autoinhibition loop is present (pink arrow). Right: Motion vectors showing conformational changes of DrmAB upon DNA binding. DrmB N-terminal half (DrmB NTH, cyan) does not make direct contacts with DNA but undergoes a large conformational change upon DNA binding, mediated by the PA of DrmA (magenta). b, EOP assay to determine the effects of V1296G or V1296W mutations to DISARM activity. c, ATPase assay measuring the rate of ATP hydrolysis by DrmAB, as monitored by increase in absorbance at $360 \mathrm{~nm}$. In the absence of DNA, no ATPase activity is monitored. In the presence of DNA stem loop lacking modification (blue), ATPase activity is observed. This is severely reduced for DNA containing three separate 5-methyl cytosine bases (orange). DNA sequences are listed in Table 2. Experiments were performed in triplicate, and the mean and standard deviation of the mean are depicted. d, Close-up views of $\operatorname{DrmA}(\mathrm{CTR})$ :DrmB interactions. e, DrmA C-terminus forms a $\beta$-sheet in trans with DrmB. $\mathbf{f}$, Effect of truncation of DrmA PA on DISARM anti-phage activity. 


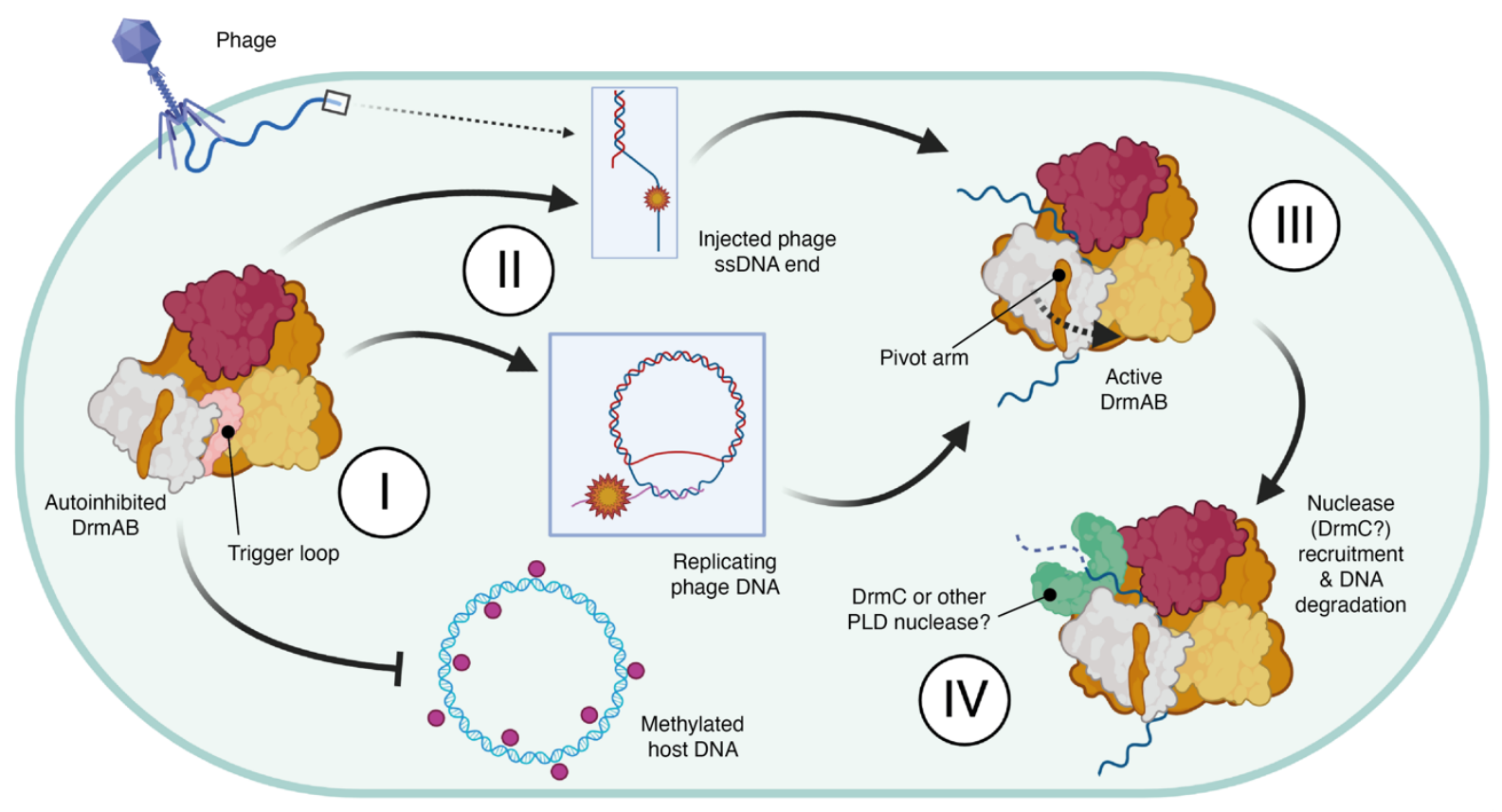

Fig. 5 I Model for anti-phage surveillance by DISARM

I. In the absence of phage infection, DrmAB complex is autoinhibited via TL. This allows the constitutive expression of the complex to enable rapid immune activation upon stimulus. II. DISARM is recruited to single-stranded 5' ovh DNA, which typically occur during initial phage DNA injection prior to genome circularization, or during rolling circle DNA replication. III. Loading of ssDNA into DrmAB dislodges TL, resulting in a conformational change and DrmAB activation. IV. Once DrmAB is active, DrmC or other nucleases may be recruited to degrade foreign DNA. DISARM may act to defend against phage at step III by loading onto phage DNA ends and physically blocking replication, and at step IV through recruiting a nuclease and degrading phage DNA. 